\title{
Identification of Gram-negative bacilli directly from positive blood culture vials
}

\author{
Correspondence \\ Thean Yen Tan \\ thean_yen_tan@cgh.com.sg
}

Received 5 May 2006

Accepted 20 November 2006

\author{
Siew Yong Ng, Lee Ling Kwang and Thean Yen Tan \\ Laboratory Medicine Services, Changi General Hospital, Singapore
}

\begin{abstract}
The provision of rapid results from positive blood cultures is important for the clinical management of septicaemia. This study tested the accuracy of direct inoculation of biochemical tests from positive blood culture vials for the identification of members of the Enterobacteriaceae and Acinetobacter species. A hundred and eighty-one samples were included in the study, with $25 \%$ subsequently excluded as a result of mixed colonial growth. The study method successfully identified 133 (98\%) isolates from 136 vials to genus level and was technically simple to perform, requiring an additional $3 \mathrm{~min}$ for the processing of each positive vial. The results of this study demonstrate that a direct inoculation method provides acceptable genus identification of Gram-negative bacilli in positive blood culture vials, with a potential saving of $24 \mathrm{~h}$ compared with traditional methods.
\end{abstract}

\section{INTRODUCTION}

The rapid provision of results for the diagnosis and treatment of bacteraemia remains one of the most important functions of a clinical microbiology laboratory. Early and appropriate treatment of bloodstream infections reduces mortality (Ibrahim et al., 2000; Micek et al., 2005). The widespread use of automated blood culture systems has reduced the time-to-detection for positive blood culture vials (Reimer et al., 1997). However, conventional methods used in the processing of positive blood culture vials usually entail a minimum delay of 2 days from the time the positive vial is detected until the identity and antimicrobial susceptibilities of the detected micro-organism are available.

Identification of Gram-negative bacilli at least to the genus level is required for the proper interpretation of susceptibility test results. Identification of the infecting bacterium may assist clinicians in determining the source of sepsis and initiating further investigations.

Multiple techniques have been described for the identification of Gram-negative bacteria from positive blood cultures. These include the direct inoculation of blood-broth aspirates onto Vitek test cards (de Cueto et al., 2004), chromogenic or fluorogenic substrates (Sepulveda et al., 1990), enzymic test strips (Edberg et al., 1975) and commercial biochemical identification kits (Malloy et al., 1983). Molecular testing methods have utilized fluorescent hybridization probes (Kempf et al., 2000) and amplification of 16S rRNA genes from positive blood culture vials (Qian et al., 2001).

Clinical microbiology laboratories face increasing manpower pressure and cost constraints (Peterson et al., 2001).
Most published methods either involve the use of expensive consumables or are too labour-intensive for routine implementation. In this study, we tested the accuracy of an identification system for members of the Enterobacteriaceae and Acinetobacter spp. based on the direct inoculation of conventional biochemical tests directly from positive blood culture vials.

\section{METHODS}

The study was carried out between January and December 2005 at an 800-bed community hospital in Singapore. Blood culture vials obtained from hospitalized patients were incubated in the Bactec 9240 system. Gram staining was performed on all vials with positive growth indices. Vials with Gram-negative bacilli only on microscopy were included in the study. When multiple vials from the same patient were detected by the system, only the initial positive vial was included in the study.

Identification of Gram-negative bacilli. Broth from the positive vial was subcultured directly onto trypticase soy agar with $5 \%$ sheep blood and onto MacConkey agar. A $0.5 \mathrm{ml}$ sample of the broth was diluted in $2.5 \mathrm{ml}$ sterile $0.85 \%$ saline. This blood/saline dilution was used to inoculate a set of commercial biochemical tubes. The tests used were a Kligler iron agar slant, a Simmons' citrate agar slant, a urea hydrolysis slant, a phenylalanine deaminase agar slant, tryptophan broth, malonate broth and an open tube of oxidative-fermentative medium. All media were incubated overnight at $35^{\circ} \mathrm{C}$ under ambient atmospheric conditions.

Following incubation, specimens with more than one colonial morphotype of Gram-negative bacilli were excluded from the study. Isolates were tested for oxidase production using a commercial reagent (Becton Dickinson) and oxidase-positive Gram-negative bacilli were excluded from further analysis. 
Biochemical tube reactions were read and interpreted according to recommended guidelines (MacFaddin, 2000). The genus of the isolate was determined according to the criteria in Table 1 (derived from data in Farmer, 2003).

Study isolates were then identified using a commercial biochemical identification system using colonies obtained from overnight growth on solid media. Members of the Enterobacteriaceae were tested using API 20E (bioMérieux), Acinetobacter spp. were tested using API 20NE (bioMérieux) and suspected Salmonella isolates were confirmed antigenically by serotyping with Salmonella antisera and biochemically using RapID ONE panels (Remel).

The genus identifications obtained by the study method and commercial identification systems were compared. Confirmatory biochemical identification by Vitek II using ID-GN panels (bioMérieux) was used to resolve study isolates with discrepant genus identification. The final genus identification was accepted if the genus identification obtained by the study method and by the initial commercial kits was concordant or if two out of the three identification systems provided similar genus identification.

Labour, times and costing. The time used to inoculate biochemical tests for the study method by different technologists was recorded on multiple occasions using a stopwatch. Test timings included the labelling and inoculation of media, interpretation of biochemical test reactions and the recording of results onto worksheets. Timing results were analysed using Microsoft EXCEL. Statistical analysis of means and standard deviation was performed using EXCEL.

The time taken to generate genus identification was calculated from the initial processing of a blood culture vial with a positive growth index until the provision of genus identification. The duration of overnight incubation was therefore included in the timing calculations.

The cost of consumables was based on prices in Singapore dollars (SGD) and converted to the British pound at the prevailing exchange rate of $\mathrm{SGD} 1=\mathfrak{E} 0.34$.

\section{RESULTS}

\section{Identification of Gram-negative bacilli}

A hundred and eighty-one positive blood culture vials were initially included in the study. Forty-five cultures were subsequently excluded when mixed colonial growth was detected following overnight subculture. The remaining 136 vials were positive for Escherichia coli $(n=42)$, Klebsiella spp. $(n=35)$, Salmonella spp. $(n=21)$, Proteus spp. $(n=11)$, Enterobacter spp. $(n=9)$, Acinetobacter spp. $(n=6)$ and other members of the Enterobacteriaceae $(n=12)$.

Genus identification was concordant with the initial testing by commercial identification systems in 131 out of the 136 isolates $(96 \%)$. The five isolates with discrepant results underwent confirmatory testing by Vitek II, following which a final accuracy of $98 \%$ for the study method (133/136 isolates) was achieved. The accuracy of genus identification for the direct testing method when compared with the reference methods is detailed in Table 2.

Two isolates were correctly identified by the direct inoculation method, but not by the initial identification

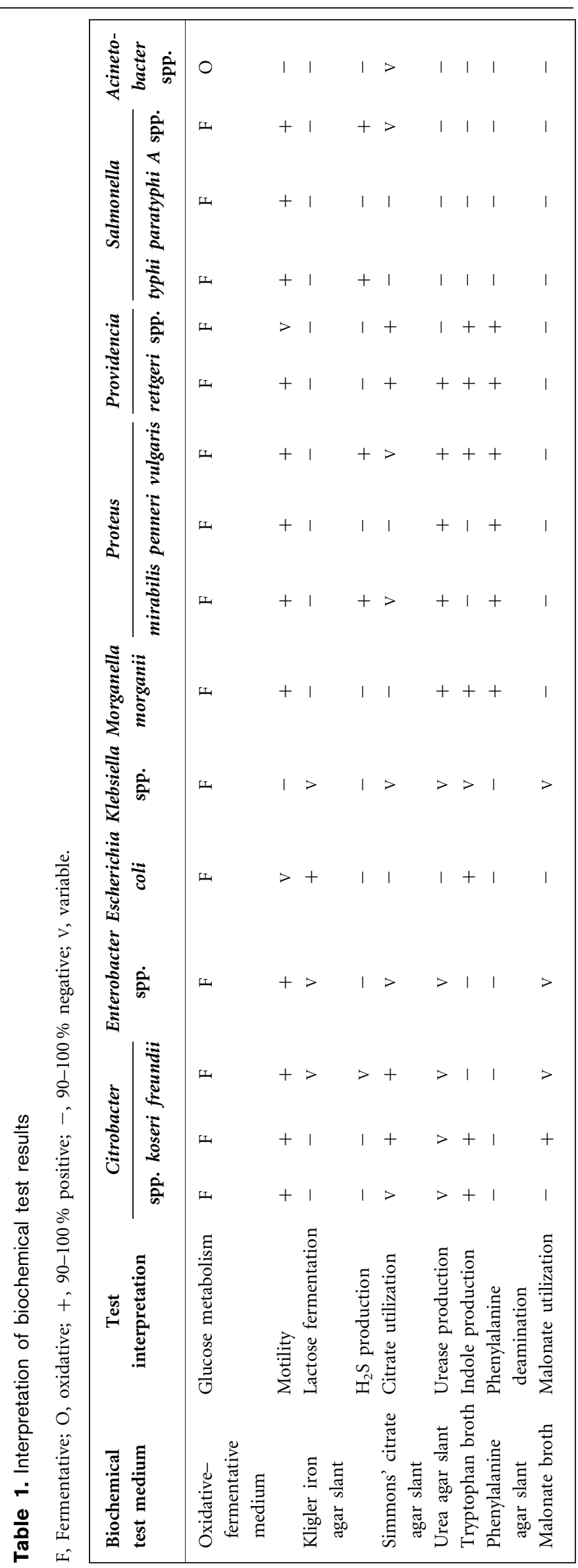


Table 2. Accuracy of the direct inoculation method compared with conventional identification methods

\begin{tabular}{|lcccc|}
\hline Organism (number) & Sensitivity (\%) & Specificity (\%) & $\begin{array}{c}\text { Positive predictive } \\
\text { value (\%) }\end{array}$ & $\begin{array}{c}\text { Negative predictive } \\
\text { value (\%) }\end{array}$ \\
\hline E. coli (42) & 98 & 100 & 100 & 99 \\
Klebsiella spp. (35) & 100 & 100 & 100 & 100 \\
Salmonella spp. (21) & 100 & 95 & 100 & 99 \\
Enterobacter spp. (9) & 100 & 100 & 100 & 100 \\
Proteus spp. (11) & 100 & 100 & 100 & 100 \\
Morganella spp. (5) & 100 & 99 & 83 & 100 \\
Providencia spp. (2) & 50 & 100 & 100 & 99 \\
Citrobacter spp. (5) & 100 & 99 & 83 & 100 \\
Acinetobacter spp. (6) & 100 & 100 & 100 & 100 \\
\hline
\end{tabular}

kit. One isolate of Morganella morganii was identified as Morganella sp. by API 20E, but with a low probability. The second isolate was identified as Providencia sp. by the study method, but misidentified as Leminorella grimontii by the identification kit. In both cases, the correct result was confirmed by Vitek 2 testing. The three isolates that were misidentified by the study method were E. coli, Providencia sp. and Salmonella sp. The E. coli isolate was initially interpreted as having pinpoint growth on the Simmons' citrate agar slant following overnight incubation. Gram staining performed on the suspected growth on the agar slant after $40 \mathrm{~h}$ incubation demonstrated an absence of bacterial cells. The Providencia sp. was misidentified by the study method as M. morganii because of the absence of growth on the Simmons' citrate agar slant following overnight incubation. Retesting of the isolate from subculture revealed obvious growth on Simmons' citrate agar, confirming the isolate as Providencia sp. The Salmonella sp. was misidentified as Citrobacter freundii as the isolate failed to agglutinate with Salmonella polyvalent $\mathrm{O}$ and $\mathrm{H}$ antigens on initial testing. Repeat serotyping following biochemical identification revealed the presence of both Salmonella $\mathrm{O}$ and $\mathrm{H}$ antigens in the isolate.

\section{Labour, timings and costing}

The mean time required to inoculate a single biochemical test from a positive blood culture vial was $22 \mathrm{~s}$ (standard deviation 5, range 18-36), whilst interpretation and recording of results took $7 \mathrm{~s}$ per test (standard deviation 1 , range 6-8) (Fig. 1). The total mean time required for inoculation of the full test panel, interpretation and recording of results was 3 min per positive blood culture vial.

The mean time taken to provide genus identification was 16-24 h for the study method, 22-31 h for Vitek 2, 40-48 h for API 20E and 64-72 h for API 20NE. For each test method used, the times included the processing time required for sample preparation.

The comparative costs of consumables including the use of

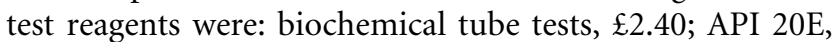
$\mathfrak{E} 5.14$; and Vitek ID-GN panel, $\mathfrak{£} 9.11$.

\section{DISCUSSION}

The results of this study demonstrate that conventional biochemical tests inoculated directly from positive blood culture vials may be used to identify Gram-negative bacilli to genus level following overnight incubation.

This study did not measure the clinical impact of providing faster identification of Gram-negative bacilli from blood cultures. A study by Trenholme et al. (1989) demonstrated that provision of direct testing results from positive blood cultures was more likely to result in changes in antibiotic

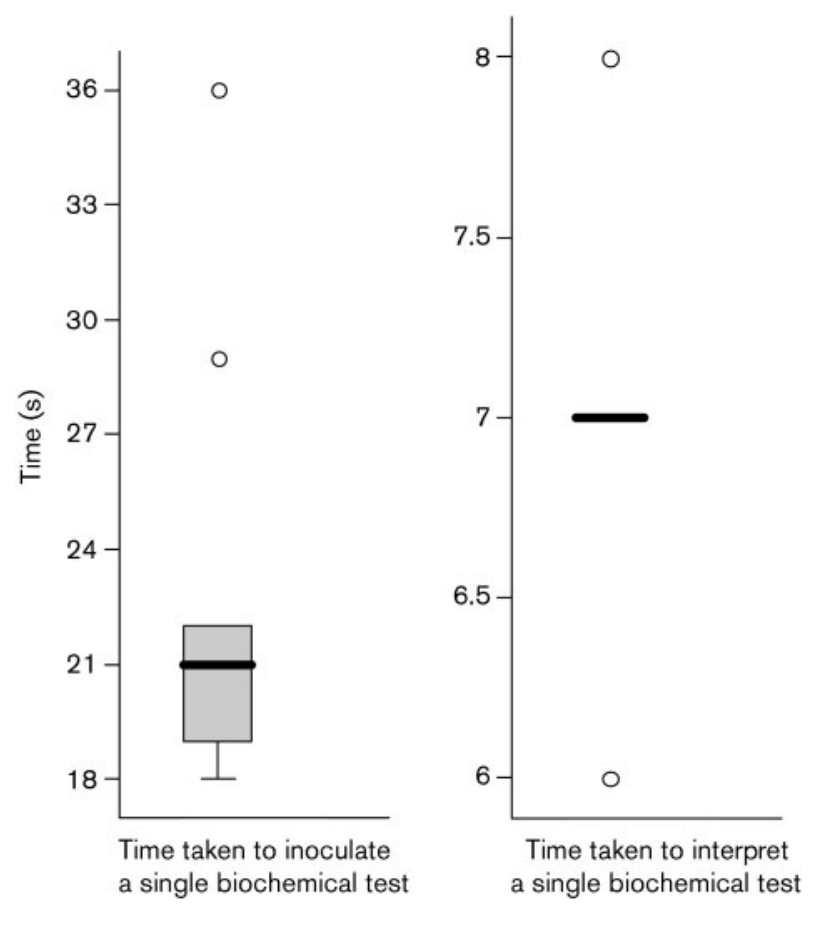

Fig. 1. Summary of the time taken to perform, read and interpret a single biochemical test. A solid line represents the median time taken, whilst the upper and lower margins of the box represent the upper and lower quartiles, respectively. Circles represent outlying values. 
therapy. Antibiotic susceptibilities of Gram-negative bacteria should be interpreted concomitant with the genus identification, as this allows improved interpretation of antibiotic susceptibilities (Livermore et al., 2001). The use of a direct inoculation method to provide genus identification may be combined with direct antibiotic susceptibility testing for earlier and more accurate reporting of positive blood cultures. Direct antibiotic susceptibility testing from positive blood culture vials has been advocated as a means of providing rapid results (Baron et al., 2005), which has been shown to be associated with clinical and financial benefits (Barenfanger et al., 1999).

The study method is less labour-intensive than other previously published methods, which usually require an additional centrifugation step (de Cueto et al., 2004; Waites et al., 1998), and may fit more easily into the workflow of a clinical microbiology laboratory.

We were unable to compare directly the full costs of the study method with those of previously published methods of direct bacterial identification from positive blood cultures, as data were lacking in the earlier studies on technologist time required to perform the reported tests. However, the costs of consumables for this method are lower than alternative methods that require the use of commercial identification systems. Financial considerations for direct testing methods are important because blood cultures with more than one bacterial species comprise $7-17 \%$ of all positive blood cultures (Ispahani et al., 1987; Pittet et al., 1997). Other studies have reported that 7-16\% of positive blood cultures with a single bacterial morphology on Gram staining are polymicrobial (Moore et al., 1981; Waites et al., 1998). In our study, $25 \%$ of blood cultures that were tested initially by the direct method had to be excluded subsequently because of mixed bacterial morphotypes following overnight culture.

The study method is only suitable for the identification of common clinical isolates of members of the Enterobacteriaceae and Acinetobacter species. Non-glucosefermenting Gram-negative bacilli, fastidious organisms such as Haemophilus influenzae and oxidase-positive glucose fermenters such as Aeromonas spp. are not identifiable by this method. E. coli and Klebsiella spp. formed the predominant isolates recovered in this study, with smaller numbers of other genera of the Enterobacteriaceae tested. Further validation of the study method may be required for bacteria other than E. coli and Klebsiella spp.

There are several limitations to the study method. Differentiation between Klebsiella spp. and Enterobacter spp. was based solely on the motility of the test isolates. Weakly motile organisms may not be visualized clearly in oxidative-fermentative tubes. Furthermore, not all Enterobacter spp. are motile (Abbott, 2003) and as such may be misidentified as Klebsiella spp. The addition of an ornithine decarboxylation test to the biochemical test series, although not performed in this study, would enable better differentiation between the two genera. Determination of a bacterium's ability to utilize citrate as a sole source of carbon energy requires $>24 \mathrm{~h}$ incubation. This may inherently result in the misinterpretation of Providencia rettgeri as $M$. morganii when the test is interpreted as negative following overnight incubation, as suggested in this study. Hydrogen sulfide-producing, non-lactose-fermenting $C$. freundii may only be differentiated from Salmonella spp. by the absence of Salmonella antigens. Although some isolates in the study were identified to species level, the study method primarily identifies Gram-negative bacilli to genus level. The accuracy of the study method is not equivalent to that obtained by commercial identification systems.

This study provides data for a method of performing direct genus identification of Gram-negative bacilli from positive blood culture vials, with acceptable levels of accuracy. Implementation of this method with concurrent direct antibiotic susceptibility testing allows for accurate interpretation of antibiotic susceptibility results, allowing provisional diagnosis and treatment to be made whilst allowing time for confirmatory test results by conventional methods. This method is suitable for routine clinical use, as it is comparatively less costly than other proposed methods and is not labour-intensive.

\section{REFERENCES}

Abbott, S. L. (2003). Klebsiella, Enterobacter, Citrobacter, Serratia, Plesiomonas and other Enterobacteriaceae. In Manual of Clinical Microbiology, 8th edn, pp. 684-696. Edited by P. R. Murray, E. J. Baron, J. H. Jorgensen, M. A. Pfaller \& R. H. Yolken, Washington, DC: American Society for Microbiology.

Barenfanger, J., Drake, C. \& Kacich, G. (1999). Clinical and financial benefits of rapid bacterial identification and antimicrobial susceptibility testing. J Clin Microbiol 37, 1415-1418.

Baron, E. J. M., Weinstein, M. P., Dunne, W. M., Jr, Yagupsky, P., Welch, D. F. \& Wilson, D. M. (2005). Cumitech 1C, Blood Cultures IV. Washington, DC: American Society for Microbiology.

de Cueto, M., Ceballos, E., Martinez-Martinez, L., Perea, E. J. \& Pascual, A. (2004). Use of positive blood cultures for direct identification and susceptibility testing with the Vitek 2 system. J Clin Microbiol 42, 3734-3738.

Edberg, S. C., Novak, M., Slater, H. \& Singer, J. M. (1975). Direct inoculation procedure for the rapid classification of bacteria from blood culture. J Clin Microbiol 2, 469-473.

Farmer, J. J., III (2003). Enterobacteriaceae: introduction and identification. In Manual of Clinical Microbiology, 8th edn, pp. 636-653. Edited by P. R. Murray, E. J. Baron, J. H. Jorgensen, M. A. Pfaller \& R. H. Yolken. Washington, DC: American Society for Microbiology.

Ibrahim, E. H., Sherman, G., Ward, S., Fraser, V. J. \& Kollef, M. H. (2000). The influence of inadequate antimicrobial treatment of bloodstream infections on patient outcomes in the ICU setting. Chest 118, 146-155.

Ispahani, P., Pearson, N. J. \& Greenwood, D. (1987). An analysis of community and hospital-acquired bacteraemia in a large teaching hospital in the United Kingdom. Q J Med 63, 427-440. 
Kempf, V. A., Trebesius, K. \& Autenrieth, I. B. (2000). Fluorescent in situ hybridization allows rapid identification of microorganisms in blood cultures. J Clin Microbiol 38, 830-838.

Livermore, D. M., Winstanley, T. G. \& Shannon, K. P. (2001). Interpretative reading: recognizing the unusual and inferring resistance mechanisms from resistance phenotypes. J Antimicrob Chemother 48 (Suppl. 1), 87-102.

MacFaddin, J. F. (2000). Biochemical Tests for Identification of Medical Bacteria. Baltimore, MD: Lippincott Williams \& Wilkins.

Malloy, P. J., Ducate, M. J. \& Schreckenberger, P. C. (1983). Comparison of four rapid methods for identification of Enterobacteriaceae from blood cultures. J Clin Microbiol 17, 493-499.

Micek, S. T., Lloyd, A. E., Ritchie, D. J., Reichley, R. M., Fraser, V. J. \& Kollef, M. H. (2005). Pseudomonas aeruginosa bloodstream infection: importance of appropriate initial antimicrobial treatment. Antimicrob Agents Chemother 49, 1306-1311.

Moore, D. F., Hamada, S. S., Marso, E. \& Martin, W. J. (1981). Rapid identification and antimicrobial susceptibility testing of Gramnegative bacilli from blood cultures by the AutoMicrobic system. J Clin Microbiol 13, 934-939.

Peterson, L. R., Hamilton, J. D., Baron, E. J., Tompkins, L. S., Miller, J. M., Wilfert, C. M., Tenover, F. C. \& Thomson, R. B., Jr (2001). Role of clinical microbiology laboratories in the management and control of infectious diseases and the delivery of health care. Clin Infect Dis 32, 605-611.
Pittet, D., Li, N., Woolson, R. F. \& Wenzel, R. P. (1997). Microbiological factors influencing the outcome of nosocomial bloodstream infections: a 6-year validated, population-based model. Clin Infect Dis 24, 1068-1078.

Qian, Q., Tang, Y.-W., Kolbert, C. P., Torgerson, C. A., Hughes, J. G., Vetter, E. A., Harmsen, W. S., Montgomery, S. O., Cockerill, F. R., III \& Persing, D. H. (2001). Direct identification of bacteria from positive blood cultures by amplification and sequencing of the $16 \mathrm{~S}$ rRNA gene: evaluation of BACTEC 9240 instrument true-positive and false-positive results. J Clin Microbiol 39, 3578-3582.

Reimer, L. G., Wilson, M. L. \& Weinstein, M. P. (1997). Update on detection of bacteremia and fungemia. Clin Microbiol Rev 10, 444-465.

Sepulveda, J. L., Stager, C. E. \& Davis, J. R. (1990). Rapid presumptive identification of Gram-negative rods directly from blood cultures by simple enzymatic tests. J Clin Microbiol 28, 177-181.

Trenholme, G. M., Kaplan, R. L., Karakusis, P. H., Stine, T., Fuhrer, J., Landau, W. \& Levin, S. (1989). Clinical impact of rapid identification and susceptibility testing of bacterial blood culture isolates. J Clin Microbiol 27, 1342-1345.

Waites, K. B., Brookings, E. S., Moser, S. A. \& Zimmer, B. L. (1998). Direct susceptibility testing with positive BacT/Alert blood cultures by using MicroScan overnight and rapid panels. J Clin Microbiol 36, 2052-2056. 